\title{
Attention to Primes Modulates Affective Priming of Pronunciation Responses
}

\author{
Jan De Houwer ${ }^{1}$ and Tom Randell ${ }^{2}$ \\ ${ }^{1}$ University of Ghent, Belgium and ${ }^{2}$ University of Southampton, Southampton, UK
}

\begin{abstract}
In studies on affective priming of pronunciation responses, two words are presented on each trial and participants are asked to read the second word out loud. Whereas some studies revealed shorter reaction times when the two words had the same valence than when they had a different valence, other studies either found no effect of affective congruence or revealed a reversed effect. In the present experiments, a significant effect of affective congruence only emerged when filler trials were presented in which the prime and target were identical and participants were instructed to attend to the primes (Experiment 2). No effects were found when participants were merely instructed to attend to or ignore the primes (Experiment 1), or when affectively incongruent filler trials were presented and participants were instructed to ignore the primes (Experiment 2).
\end{abstract}

Key words: affective priming, automatic attitude activation, word reading

In a typical affective priming study, two words are presented in each trial that either have the same valence (e.g., HOLIDAY - SINCERE) or a different valence (e.g., HOLIDAY -ANGRY). Participants are asked to determine the valence of the second word (i.e., the target) but to ignore the first word (i.e., the prime). Most often, an affective priming effect is observed; that is, faster responses when the target is preceded by an affectively related prime than when the prime has a different valence. These findings have been taken as evidence for the hypothesis that attitudes can be activated automatically in the sense of being involuntary, fast, and unconscious (for reviews see Fazio, 2001; Klauer \& Musch, in press).

Researchers have also examined whether affective priming occurs when participants are asked to read target words out loud. If one can demonstrate that the match between the valence of the prime and the

The research described here was supported by a grant from the ESRC (R000 22 3373) to Jan De Houwer. Experiments 1 and 2 were conducted while Jan De Houwer was a lecturer at the University of Southampton. We thank Jack Glaser and John Bargh for their helpful comments on an earlier version of this paper, and Frank Baeyens for conducting the power analyses. valence of the target influences the time needed to read the targets, this would demonstrate that the attitude toward the prime was activated even though participants did not have the intentional goal of evaluating stimuli in their environment. Moreover, affective priming of pronunciation responses could provide a very unobtrusive measure of attitudes. For instance, if a prime facilitates the reading of positive rather than negative words, one could infer that participants have a positive attitude toward the prime (e.g., Fazio, Jackson, Dunton, \& Williams, 1995; Fazio, Sanbonmatsu, Powell, \& Kardes, 1986; Glaser \& Banaji, 1999). This measure could provide unique information. For instance, it is possible that some but not all attitudes will influence behavior when there is no explicit goal to evaluate. Affective priming tasks in which participants are asked to evaluate targets will not be able to pick up these differences.

Unfortunately, the evidence regarding affective priming of pronunciation responses is equivocal. The first studies in which this phenomenon was examined revealed large effects. In these studies, reaction times were faster on trials with affectively congruent prime-target pairs than on trials with affectively incongruent pairs, regardless of whether primes had a 
clear positive or negative meaning (strong primes) or were only slightly positive or negative (weak primes) (Bargh, Chaiken, Raymond, \& Hymes, 1996; Hermans, De Houwer, \& Eelen, 1994). In contrast, Glaser and Banaji (1999) observed faster responses on affectively incongruent than on affectively congruent trials with strong primes. With weak primes, responses were sometimes faster on congruent than on incongruent trials but in other experiments no difference was found. Finally, Klauer and Musch (2001) conducted an impressive series of studies, none of which revealed any evidence for either standard or reversed affective priming effects in the pronunciation task.

On the basis of this mixed pattern of results, one might be tempted to conclude that affective priming of pronunciation responses is not a genuine phenomenon. However, this conclusion does not seem justified. Bargh et al. (1996) obtained strong effects in three consecutive experiments. Glaser and Banaji (1999) observed significant reverse priming effects in seven consecutive experiments. This consistency of results within labs suggests that affective priming of pronunciation responses is a genuine phenomenon, but that its magnitude and direction depends upon certain procedural variables. More needs to be known about these variables before affective priming of pronunciation responses can be used as a reliable tool to study and measure automatic attitude activation.

Glaser and Banaji (1999) hypothesized that reversed priming effects might occur because participants automatically counteract for the influence of the primes. ${ }^{1}$ The mere fact that at the start of the experiment participants consciously set a goal to avoid or counteract any influence of the irrelevant primes could be sufficient for this goal to operate at an automatic level during the experiment. Recent research confirms that automatic motivation can operate in this way to influence judgments and behavior (e.g., Bargh \& Chartrand, 1999). Differences with regard to how participants deal with the primes might account for the different results obtained by Glaser and Banaji (1999) and Bargh et al. (1996).

\footnotetext{
1 Motivation to correct the influence of the primes is related to motivation to respond accurately. Glaser (in press) recently found that affective priming of pronunciation responses tended to be weaker (but not reversed) when accuracy motivation was high (i.e., when participants were informed that trials on which an incorrect response was made would be repeated and that it would thus be in their benefit to respond accurately) than when it was low (i.e., when participants were just asked to read the second word out loud). Note, however, that a high accuracy motivation does not necessarily imply that participants try to counteract the influence of primes, nor does a low accuracy motivation imply that participants attend to primes.
}

Whereas Bargh et al. (1996, p. 110) merely asked participants to pronounce the second word and gave no instructions about how to deal with the primes, Glaser and Banaji (1999, p. 674) informed participants that the experiment "was designed to measure how well people can make responses in the presence of distracting stimuli."

To test whether orientation to the primes influences affective priming of pronunciation responses, in Experiment 1, we randomly assigned participants to two groups that differed with regard to the way they were instructed to deal with the primes. The first group was informed that the primes were meant to disrupt the task of reading the targets and were instructed to try to counteract the influence of the primes as much as possible. Participants in the second group were told that the primes could help them perform the task and were instructed to utilize the primes as much as possible. Both groups then completed the same priming phase during which they read the targets out loud. On the basis of the arguments put forward by Glaser and Banaji (1999), one can predict that a smaller (or even reversed) priming effect will emerge in the first group than in the second group.

\section{Experiment 1}

\section{Method}

\section{Participants}

Fifty-two undergraduate psychology students at the University of Southampton participated for partial fulfillment of course requirements. All were native English speakers.

\section{Stimuli}

The 20 targets were identical to those used by Bargh et al. (1996, Experiment 2). As primes, we selected eight positive nouns, eight negative nouns, and four neutral letter strings. Four of the positive and negative primes were the same as the strong primes used by Bargh et al. (1996, Experiment 2). The other four positive (summer, birthday, gift, music) and four negative (war, bombs, crime, hatred) primes were selected on the basis of the normative ratings reported by Bargh, Chaiken, Govender, and Pratto (1992), using the same criteria that Bargh et al. (1996) used to select their strong primes (see Appendix A). A further 10 adjectives (primes) and 10 nouns (targets) were only presented on the practice trials.

All words were written in white upper-case letters on a black background and were presented on a 14- 
inch VGA screen connected to an IBM compatible 486 computer. Each letter was $7 \mathrm{~mm}$ high and $5 \mathrm{~mm}$ wide. Presentations were controlled by a Turbo Pascal 5.0 program, which operated in graphics mode. Participants were seated in front of the computer screen at a distance of approximately $40 \mathrm{~cm}$. Verbal responses were registered using a voice key that generated a signal that stopped a highly accurate (better than $1 \mathrm{~ms}$ ) Turbo Pascal Timer (Bovens \& Brysbaert, 1990).

\section{Procedure}

Instructions were presented on the computer screen. Participants were first informed that on each trial two words would appear on the screen consecutively. They were asked to read the second word out loud as quickly as possible without making too many errors. Those who were assigned to the first condition (attend to primes) were then given the following instructions:

Although you do not have to read the first word out loud, it is crucial that you do pay attention to the first word. The first word can help you read the second word, so it is essential that you open your mind to the first word as much as possible.

Participants who were assigned to the second condition (ignore primes) instead received the following instructions:

You should not read the first word out loud. Rather, it is crucial that you try to ignore the first word as much as possible. The first word can distract you from reading the second word, so it is essential that you try to mentally counteract the disruptive influence of the first word as much as possible without diverting your eyes.

The practice primes were randomly assigned to the practice targets and the 10 resulting prime-target pairs were presented in a random order. For each of five experimental blocks, each prime was assigned to one randomly selected target in such a way that there were eight affectively congruent, eight affectively incongruent, and four control trials. In each block, the resulting 20 prime-target pairs were presented in a random order with the restriction that the valence of the target could not be the same on more than four consecutive trials. The sequence of events on each practice and each experimental trial was as follows: A fixation cross for $500 \mathrm{~ms}$, the prime for $200 \mathrm{~ms}$, a blank screen for $100 \mathrm{~ms}$, and the target until a response was registered or $3000 \mathrm{~ms}$ had elapsed. The experimenter then pressed one of three keys to indicate whether the voice key accurately registered the response and whether the response was correct. The next trial started $4000 \mathrm{~ms}$ after the experimenter entered the code. Blocks were separated by a user-terminated pause. During this pause, participants in the attend prime condition were shown the message "Remember to attend the first word," whereas participants in the ignore prime condition saw the message "Remember to ignore the distracting first word." After all prime-target pairs were presented, participants were given a recognition test during which the 20 experimental primes and 20 distractors were presented one by one. The distractors were 10 positive and 10 negative nouns that were not presented earlier during the experiment. Participants were told that half of the words had been previously presented and were asked to decide, for each word, whether it had previously been presented or not.

\section{Results and Discussion}

For each participant, we calculated the mean reaction time on each of the four types of experimental trials ( 2 prime valences $\times 2$ target valences). Trials on which the voice key failed to accurately register the response $(3.80 \%)$, an incorrect response was given $(0.85 \%)$, or the reaction time was shorter than 150 $\mathrm{ms}$ or longer than $1500 \mathrm{~ms}(0.09 \%)$ were discarded. In accordance with Bargh et al. (1996), raw latencies were $\log$ transformed before they were submitted to an analysis of variance. ${ }^{2}$ The untransformed means can be found in Table 1 .

A Condition (attend to or ignore primes) $\times$ Prime Valence (positive or negative) $\times$ Target Valence (positive or negative) ANOVA with repeated measures on the last two variables only revealed a main effect of target valence, $F(1,50)=8.67, p<.001$. As was the case in the studies of Bargh et al. (1996), reaction times were shorter for negative targets than for positive targets. Neither the interaction between prime valence and target valence, nor the three-way interaction was significant, $F \mathrm{~s}<1$, all other $F_{\mathrm{s}}<1$. We then conducted two Prime Valence $\times$ Target Valence ANOVAs to examine affective priming in each condition separately. In both conditions, affective priming, as indexed by the Prime Valence $\times$ Target Valence interaction, was not significant, $F \mathrm{~s}<1$.

As a manipulation check, we calculated the proportion of correctly recognized primes during the post-experimental recognition task and deducted from this the proportion of incorrectly recognized distractors. This recognition performance measure had a higher value in the attend prime condition $(M=$ $.83, S D=.09)$ than in the ignore prime condition

2 In this and all other experiments, we also analyzed the untransformed mean reaction times. All these analyses revealed virtually the same results and led to the same conclusions as the analyses of the log transformed mean reaction times. 
Table 1. Mean Reaction Times in ms (SD in Parentheses) as a Function of Condition, Prime Valence, and Target Valence in Experiments 1 and 2

\begin{tabular}{|c|c|c|c|}
\hline \multicolumn{4}{|c|}{ Prime valence } \\
\hline $\begin{array}{l}\text { Condition } \\
\text { and target valence }\end{array}$ & Positive & Negative & PE \\
\hline \multicolumn{4}{|c|}{ Experiment 1} \\
\hline Attend to primes & & & \\
\hline Positive & $522(93)$ & $521(97)$ & \\
\hline Negative & $511(98)$ & $512(101)$ & $-1(31)$ \\
\hline \multicolumn{4}{|l|}{ Ignore primes } \\
\hline Positive & $514(67)$ & $520(76)$ & \\
\hline Negative & $506(65)$ & $508(75)$ & $2(23)$ \\
\hline \multicolumn{4}{|c|}{ Experiment 2} \\
\hline Attend to primes & & & \\
\hline Positive & $516(69)$ & $537(78)$ & \\
\hline Negative & $524(73)$ & $522(78)$ & $12 *(32)$ \\
\hline \multicolumn{4}{|l|}{ Ignore Primes } \\
\hline Positive & $541(93)$ & $533(86)$ & \\
\hline Negative & $537(94)$ & $534(90)$ & $-3(31)$ \\
\hline
\end{tabular}

$* p<.05$

Note. $\mathrm{PE}=$ Priming Effect, that is mean reaction time on incongruent trials (positive prime, negative target; negative prime, positive target) minus mean reaction time on congruent trials (positive prime, positive target; negative prime, positive target).

$(M=.72, S D=.20), t(36.32)=-2.55, p<.05$, which suggests that participants in the attend prime condition indeed paid more attention to the primes.

The present study revealed only small, nonsignificant priming effects, regardless of whether participants were instructed to attend to the primes or were asked to actively counteract the influence of the primes. Power analyses (see Hendrickx, De Houwer, Baeyens, Eelen, \& Van Avermaet, 1997, Appendix) nevertheless showed that our tests had adequate power to detect a large $(f=0.80$; power of 1.00 for the total group and .97 for each condition separately) or medium sized $(f=0.50$; power of .94 for the total group and .69 for each condition separately) priming effect. Our results stand in sharp contrast to those reported by Bargh et al. (1996), who did obtain large priming effects, and to the results of Glaser and Banaji (1999), who consistently observed reversed priming effects for strong primes. Rather, the results support the findings of Klauer and Musch (2001) who also repeatedly failed to observe affective priming of pronunciation responses.

\section{Experiment 2}

Apart from instructing half of the participants to attend to the primes and the other half to actively counteract the effect of the primes, in Experiment 2 we included filler trials that further encouraged participants to adopt the instructed approach. In the attend prime condition, the prime and target were identical on all filler trials (e.g., HAPPY-HAPPY). Because of the high percentage of such filler trials (i.e., $67 \%$ ), participants' performance in reading the target words would indeed benefit from an increased attention to the primes. In the ignore prime condition, however, all filler trials were affectively incongruent trials. Moreover, the words that were presented on those filler trials functioned as primes and as targets an equal number of times. Because filler primes were thus potential targets, participants should be more inclined to actively ignore the primes. The experimental trials, which consisted of 20 affectively congruent and 20 affectively incongruent trials, were identical in both conditions.

\section{Method}

\section{Participants}

Sixty-four students at the University of Southampton participated. Nonpsychology students received £6, psychology students either received course credits or $£ 6$. None had participated in Experiment 1 and all were native English speakers.

\section{Stimuli}

The targets on the experimental trials were the same as in Experiment 1 except for the word AVERSIVE, which was replaced by the word DREADFUL because the former word was unfamiliar to a number of our British participants. The experimental primes were also the same as in Experiment 1 except that the four neutral letter strings were replaced by two positive (love, flower) and two negative (slime, corpse) words (see Appendix B). On the filler trials, 10 additional positive and 10 additional negative adjectives were presented as targets and primes. Finally, during the practice trials, five other positive and five other negative adjectives functioned as primes and targets. The apparatus that was used to present the stimuli and record the responses was the same as in Experiment 1, as was the way in which the stimuli were presented. 


\section{Procedure}

Only the differences with Experiment 1 will be described. First, the instructions were phrased slightly differently. In the attend prime condition, instructions said that:

Although you do not have to read the first word out loud, it is crucial that you do pay attention to the first word. On many trials, the first word will be the same as the second word. So if you always attend to the first word, this will help you read the second word more quickly.

In the ignore prime condition, these instructions were replaced by the following sentences:

You should not read the first word out loud. Rather, it is crucial that you try to ignore the first word as much as possible. The first word will always differ from the second word and will thus always distract you from reading the second word quickly. Therefore, try to mentally counteract the disruptive influence of the first word as much as possible without diverting your eyes.

A second difference was that each experimental prime and target was only presented twice, once as part of an affectively congruent prime-target pair and once as part of an affectively incongruent pair. Within these restrictions, primes were randomly assigned to targets. Each filler was presented four times as a target and four times as a prime. In the attend prime condition, the filler prime and target were always identical. In the ignore prime condition, filler primes were randomly assigned to filler targets with the restriction that a prime always had a different valence than the target to which it was assigned. The 10 practice pairs were the same for all participants who were assigned to the same condition. In the attend prime condition, there were eight practice trials on which the prime was identical to the target. In the ignore prime condition, those practice prime - target pairs were rearranged to form eight affectively incongruent pairs. In both conditions, one affectively congruent and one affectively incongruent practice prime-target were also presented. All practice stimuli differed from the stimuli used on the experimental and filler trials.

Practice trials were presented in a random order. After a break, 20 filler trials (all filler primes and targets once) were presented, followed by 60 filler trials intermixed with the 40 experimental trials. Participants could take a break after every 30 trials. During this break, participants only saw the following instruction: "Press <enter > to continue." The first trial after each break was always a filler trial. Apart from the above restrictions and the restriction that the valence of the target could not be the same on more than four consecutive trials, the order of the filler and experimental trials was randomized for each participant separately. There was no recognition task at the end of the experiment.

\section{Results}

The reaction times on experimental trials were analyzed in the same way as in Experiment 1. Trials on which a voice key failure occurred (3.8\%), an incorrect response was given $(1.58 \%)$, or reaction times were shorter than $100 \mathrm{~ms}$ or longer than $1500 \mathrm{~ms}$ $(0.04 \%)$ were discarded. The relevant means can be found in Table 1.

The Condition $\times$ Prime Valence $\times$ Target Valence ANOVA revealed a marginally significant three-way interaction, $F(1,62)=3.40, p=.07$, all other $F \mathrm{~s}<$ 1.79. Separate analyses showed that the Prime Valence $\times$ Target Valence interaction was significant in the attend prime condition, $F(1,31)=5.05, p=.03$, but not in the ignore prime condition, $F<1$.

\section{Discussion}

As predicted, reaction times were faster on affectively congruent than on affectively incongruent trials when participants were instructed to attend to the primes and when attention to primes was encouraged by including filler trials on which the prime and target were identical. Note that this effect occurred even though the prime and target were always different on the congruent and incongruent trials. In contrast, no effect was found in the ignore prime condition. It is, however, unclear whether the significant priming effect in the attend prime condition was due to the nature of the filler trials, the instruction to attend the primes, or a combination of both. One could argue that because of the large number of filler trials on which the prime and target were identical, participants spontaneously oriented their attention to the primes, regardless of explicit instructions on how to deal with the primes. Similar effects of the proportion of trials on which prime (or distractor) and target match with regard to a task relevant stimulus feature have been found in Stroop studies (e.g., Logan, 1980) and affective priming studies with evaluation responses (e.g., Klauer, Roßnagel, \& Musch, 1997).

To shed some light on this issue, we conducted an additional experiment that was identical to the attend prime condition of Experiment 2, but we deleted the instruction that urged participants to attend to the primes (see above). That is, participants were simply asked to read the second word on each trial. Sixteen first year psychology students at the University of Southampton participated. The data were analyzed in the same way as the data of attend prime condition of Experiment 2. Only a small, nonsignificant affective priming effect was found (mean affective priming effect of $5 \mathrm{~ms}, S D=38, F<1$ ). Because we did not find a significant priming effect when only filler trials with identical primes and targets were present, or 
when only instructions to attend the primes were given with no filler trials (attend prime condition of Experiment 1), but did find an effect when both filler trials and instructions were presented (attend prime condition of Experiment 2), it appears to be the case that the latter result was at least partly due to the combination of filler trials and instructions. ${ }^{3}$ Note, however, that this conclusion needs to be treated with some caution because of the small number of participants in the additional experiment and because the conclusion is based on a comparison between experiments. But also note that regardless of the precise explanation of the results of Experiment 2, the results do suggest that the degree to which participants (strategically or more spontaneously) pay attention to the primes modulates affective priming of pronunciation responses.

Finally, a reviewer of this article pointed out that a target might be easier to pronounce when preceded by a prime that starts with a similar phoneme (e.g., DISEASE-DISTRESSING), especially when participants are encouraged to attend to the primes. This might be responsible for the observed effects of affective congruence, provided that a target was more likely to start with the same phoneme as an affectively congruent prime than an affectively incongruent prime. Appendix B, however, shows that there were only very few primes and targets that started with the same phoneme and this number was virtually the same for affectively congruent primes and targets and affectively incongruent primes and targets. Nevertheless, a substantial number of our negative primes and negative targets started with a plosive sound (e.g., TERRIBLE), whereas most of our positive primes and positive targets started with a nonplosive sound (e.g., APPEALING). Assuming

\footnotetext{
3 At the end of the additional experiment, participants were asked to express how much they intentionally paid attention to the first word in order to speed up their responses. They could do so by giving a number between -100 (tried very hard to ignore) and +100 (tried very hard to attend). Afterwards, they also rated how much they thought they actually attended or ignored the primes (intentional or not), again by giving a number between -100 (actually ignored very much) to +100 (actually attended very much). Participants gave a mean rating of -16.56 $(S D=61.72)$ in response to the first question, suggesting that they tended to try to ignore the primes. The mean rating for the second question was $30.94(S D=58.86)$, suggesting that the participants thought that they actually did pay attention to the primes. Assuming that participants in the attend prime condition of Experiment 2 did intentionally try to attend the primes (as they were instructed to do), one might conclude that the intention to attend to the primes (and thus the instructions related to this intention) did indeed play an important role in the emergence of the priming effect in the attend prime condition of Experiment 2 .
}

that plosive (nonplosive) primes facilitate the pronunciation of plosive (nonplosive) targets, this confound might be problematic. To further examine the effect of the match between the type of first phoneme (plosive or nonplosive), we calculated for each participant the mean log-transformed reaction time on trials with a plosive prime and target, a nonplosive prime and plosive target, a plosive prime and nonplosive target, and a nonplosive prime and nonplosive target. A Condition (attend prime or ignore prime) $\times$ Type of Prime (plosive or nonplosive) $\times$ Type of Target (plosive or nonplosive) ANOVA showed that neither the interaction between type of prime and type of target, nor the three-way interaction was significant, $F \mathrm{~s}<1$. A priori contrasts showed that there was no sign of an effect of type of prime, neither for plosive targets (mean for plosive primes $=513 \mathrm{~ms}$; mean for nonplosive primes $=508 \mathrm{~ms}, t<1$ ), nor for nonplosive targets (mean for plosive primes $=$ $545 \mathrm{~ms}$; mean for non-plosive primes $=542 \mathrm{~ms}, t<$ 1). These analyses thus fail to support the alternative explanation of our results in terms of phoneme match.

\section{General Discussion}

Affective priming of pronunciation responses could potentially be used to measure and study attitudes in interesting new ways. Unfortunately, such priming effects tend to be unreliable and even the direction of the effects can differ between studies. The present results suggest that the extent to which participants pay attention to the primes modulates the strength of affective priming effects in the pronunciation task. This observation is important in its own right because it allows us to better understand the conditions under which affective priming of pronunciation responses will occur. Moreover, our results might also shed some light on the existing literature. One could argue that the differences between the results of previous studies were partly due to differences in the degree to which participants paid attention to primes. Although no previous studies have included the kind of directive instructions and filler trials that were used in the present experiments, it is possible that other, less salient, aspects of the procedure used in those studies also had an impact on participants' orientation to the primes.

Although a significant standard priming effect (i.e., faster responding on congruent trials) was observed in the attend prime condition of Experiment 2 , no priming effects were found in the other conditions. This repeated failure to observe priming effects is remarkable given that the procedure used in our studies was similar to the procedure that was successfully employed by Bargh et al. (1996). One im- 
portant difference with the original studies of Bargh et al., however, was that we explicitly told the participants how they should deal with the primes. The instructions used by Bargh et al., on the other hand, were specifically designed to discourage participants from processing the primes in a strategic way (i.e., participants were simply asked to read the second word). It is possible that priming effects in the pronunciation task are more likely to emerge when participants do not exert any effort to process primes in a certain way (i.e., either attend or counteract). ${ }^{4}$ Note, however, that this hypothesis is similar to the conclusion that we reached in that it also emphasizes the role of orientation to the primes. It is also worth mentioning that we conducted an experiment that was identical to Experiment 1 except that only three blocks of trials were given and that the instructions of Bargh et al. (1996) were used (i.e., each of the 20 participants was merely asked to read the second word on every trial). Despite the lack of any explicit instruction on how to deal with the primes, a nonsignificant priming effect of $6 \mathrm{~ms}, S D=32, t<1$, was observed. We also know of several other almost identical replications of the studies of Bargh et al. (1996) that revealed no significant priming effects (Klauer \& Musch, 2001, Experiment 5; Spruyt, 1999; also see the additional experiment reported in the discussion of Experiment 2). In fact, it was failures such as these that prompted us to look for moderating variables.

We also obtained little evidence for reversed priming effects, such as those reported earlier by Glaser and Banaji (1999). These authors argued that reverse priming effects might occur when participants automatically counteract for the influence of the primes. Based on this argument, we expected a reverse priming effect in the ignore prime conditions of the present experiments. Although our results do not support the hypothesis put forward by Glaser and Banaji, one could argue that our instructions were not explicit enough to induce an automatic tendency to counteract for the influence of the primes. For instance, the fact that during the break between each experimental block, participants were asked to ignore the primes rather than to counteract the influence of the primes, might have put a larger emphasis on the fact that primes had to simply be ignored rather than corrected for. Further research is thus needed.

Although we most often found little evidence for affective priming in the present studies, a significant effect was observed in the attend prime condition of Experiment 2. This finding confirms that affective priming of pronunciation responses can be observed under certain conditions and supports the hypothesis

\footnotetext{
4 We thank John Bargh for drawing our attention to this argument.
}

that attitudes can be activated automatically, even when participants do not have the conscious, intentional goal of evaluating stimuli in their environment. The present results also suggest ways in which affective priming of pronunciation responses can be transformed into a reliable measure of attitudes. If one wants to use this task as an indirect measure of attitudes, one can increase the magnitude of the effects by adding filler trials on which the prime and target are identical. Previous research has demonstrated that effects are also stronger when targets are difficult to read (De Houwer, Hermans, \& Spruyt, 2001) and when pictures rather than words are used as primes and targets (Spruyt, Hermans, De Houwer, \& Eelen, 2001). It would be interesting to investigate whether the effects of attention to primes, target legibility, and target modality are additive. If this is the case, it should be possible to develop a procedure that produces the kind of strong and reliable effects that one needs in order to use affective priming of pronunciation responses as an indirect measure of attitudes.

\section{References}

Bargh, J. A., Chaiken, S., Govender, R., \& Pratto, F. (1992). The generality of the attitude activation effect. Journal of Personality and Social Psychology, 62, $893-912$.

Bargh, J. A., Chaiken, S., Raymond, P., \& Hymes, C. (1996). The automatic evaluation effect: Unconditional automatic activation with a pronunciation task. Journal of Experimental Social Psychology, 32, 104-128.

Bargh, J. A., \& Chartrand, T. L. (1999). The unbearable automaticity of being. American Psychologist, 54, $462-479$

Bovens, N., \& Brysbaert, M. (1990). IBM PC/XT/AT and $\mathrm{PS} / 2$ Turbo Pascal timing with extended resolution. Behavior Research Methods, Instruments, and Computers, 22, 332-334.

De Houwer, J., Hermans, D., \& Spruyt, A. (2001). Affective priming of pronunciation responses: Effects of target degradation. Journal of Experimental Social Psychology, 37, 85-91.

Fazio, R. H. (2001). On the automatic activation of associated evaluations. Cognition and Emotion, 15, 115142.

Fazio, R. H., Jackson, J. R., Dunton, B. C., \& Williams, C. J. (1995). Variability in automatic activation as an unobtrusive measure of racial attitudes: A bona fide pipeline? Journal of Personality and Social Psychology, 69, 1013-1027.

Fazio, R. H., Sanbonmatsu, D. M., Powell, M. C., \& Kardes, F. R. (1986). On the automatic activation of attitudes. Journal of Personality and Social Psychology, 50, 229-238.

Glaser, J. (in press). Reverse priming: Implications for the (un)conditionality of automatic evaluation. In $\mathrm{J}$. Musch \& K. C. Klauer (Eds.), The psychology of evaluation: Affective processes in cognition and emotion. Mahwah, NJ: Lawrence Erlbaum. 
Glaser, J., \& Banaji, M. R. (1999). When fair is foul and foul is fair: Reverse priming in automatic evaluation. Journal of Personality and Social Psychology, 77, $669-687$.

Hendrickx, H., De Houwer, J., Baeyens, F., Eelen, P., \& Van Avermaet, E. (1997). Hidden Covariation Detection might be very hidden indeed. Journal of Experimental Psychology: Learning, Memory, and Cognition, 23, 201-220.

Hermans, D., De Houwer, J., \& Eelen, P. (1994). The affective priming effect: Automatic activation of evaluative information in memory. Cognition and Emotion, 8, $515-533$.

Klauer, K. C., \& Musch, J. (in press). Affective priming: Findings and theories. In J. Musch \& K. C. Klauer (Eds.), The psychology of evaluation: Affective processes in cognition and emotion. Mahwah, NJ: Lawrence Erlbaum.

Klauer, K. C., \& Musch, J. (2001). Does sunshine prime loyal? Affective priming in the naming task. Quarterly Journal of Experimental Psychology, 54A, 727-751.

Klauer, K. C., Roßnagel, C., \& Musch, J. (1997). Listcontext effects in evaluative priming. Journal of Experimental Psychology: Learning, Memory, and Cognition, 23, 246-255.

Logan, G. D. (1980). Attention and automaticity in Stroop and priming tasks: Theory and data. Cognitive Psychology, 12, 523-553.

Spruyt, A. (1999). Automatische stimulusevaluatie. Affectieve primingeffecten in de uitspraaktaak als functie van orthographische diepte, degradatie en taalvertrouwdheid [Automatic stimulus evaluation. Affective priming effects as a function of orthographical depth, degradation and language familiarity]. Unpublished master's thesis, University of Leuven, Leuven, Belgium.

Spruyt, A., Hermans, D., De Houwer, J., \& Eelen, P. (2001). Affective priming in a picture naming task. Manuscript submitted for publication.

Jan De Houwer

Department of Psychology

University of Ghent

Henri Dunantlaan 2

B-9000 Ghent

Belgium

Tel.: +32 2646445

Fax: +32 2646489

E-mail: Jan.DeHouwer@rug.ac.be

\section{Appendix A}

\section{Targets and Primes Presented in Experiment 1}

Positive Targets: TERRIFIC, OUTSTANDING, FASCINATING, ATTRACTIVE, REFRESHING, SATISFYING, DELIGHTFUL, APPEALING, PLEASANT, ENJOYABLE

Negative Targets: AVERSIVE, GRUESOME, REPULSIVE, FRIGHTFUL, HORRIBLE, SICKENING, DISTRESSING, TERRIBLE, DISGUSTING, OFFENSIVE

Positive Primes: FRIEND, DANCING, FLOWERS, HOLIDAY, SUMMER, BIRTHDAY, GIFT, MUSIC

Negative Primes: CANCER, DEATH, FUNERAL, DISEASE, WAR, BOMBS, CRIME, HATRED

Neutral Primes: DDD, FFF, MMM, RRR

\section{Appendix B}

\section{Targets and Primes Presented in Experiment 2}

Positive Targets: TERRIFIC*, OUTSTANDING, FASCINATING, ATTRACTIVE, REFRESHING, SATISFYING, DELIGHTFUL*, APPEALING, PLEASANT*, ENJOYABLE

Negative Targets: DREADFUL*, GRUESOME*, REPULSIVE, FRIGHTFUL, HORRIBLE, SICKENING，DISTRESSING*，TERRIBLE*，DISGUSTING*, OFFENSIVE

Positive Primes: FRIEND, DANCING*, FLOWERS, HOLIDAY, SUMMER, BIRTHDAY*, GIFT*, MUSIC, LOVE, FLOWER

Negative Primes: CANCER*, DEATH*, FUNERAL, DISEASE*, WAR, BOMBS*, CRIME*, HATRED, SLIME, CORPSE*

Positive Filler Targets: BEAUTIFUL, GENEROUS, CHEERFUL, PEACEFUL, TRANQUIL, BRILLIANT, THOUGHTFUL, FAMOUS, GENUINE, CHARMING

Negative Filler Targets: DEPRESSED, VULGAR, CORRUPT, DISAPPOINTED, INCOMPETENT, COWARDLY, GUILTY, INFERIOR, GREEDY, ASHAMED

Note. Words marked with an * were classified as starting with a plosive sound. 